\title{
氯化铯催化羧酸钠(钾)与 $\alpha$-氯代乙酸酯及溴(氯)代烃反应
}

\author{
刘文奇 $a$ 奇建永 ${ }^{b}$ 陈锦杨 ${ }^{b}$ 李宁波 ${ }^{b}$ 邱仁华 $b$ 许新华*, $b$ \\ ( ${ }^{a}$ 湘南学院湘南稀贵金属化合物及其应用湖南省重点实验＼cjkstart湘州 423000) \\ ( 湖南大学化学化工学院 长沙 410082)
}

\begin{abstract}
摘要 以 DMF 为溶剂, 在催化量氯化铯存在下, 羧酸钠(钾)与 $\alpha$-氯代乙酸酯在室温下反应及其与澳(氯)代烃在 $60{ }^{\circ} \mathrm{C}$ 反 应, 分别形成对应的羧酸 $(\alpha-$ 烷氧甲酰)甲酯与羧酸酯, 产率 $90 \%$ 以上, 讨论了催化机理.
\end{abstract}

关键词 氯化铯; 羧酸钠(钾); $\alpha$-氯代乙酸酯; 酯化反应

\section{Cesium Chloride Catalysized the Reaction of Sodium (Potassium) Carboxylates with Alkyl Bromides (Chlorides)}

\author{
Liu, Wenqi ${ }^{a}$ \\ Qi, Jianyong ${ }^{b}$ \\ Chen, Jinyang ${ }^{b}$ \\ Li, Ningbo ${ }^{b}$ \\ Qui, Renhua ${ }^{b}$ \\ $\mathrm{Xu}, \mathrm{Xinhua}^{*, b}$ \\ $\left({ }^{a}\right.$ Hunan Provincial Key Laboratory of Xiangnan Rare-Precious Metals Compounds Research and \\ Application, Xiangnan University, Chenzhou 42300) \\ $\left({ }^{b}\right.$ College of Chemistry Chemical and Engineering, Hunan University, Changsha 410082)
}

\begin{abstract}
In the presence of catalytic amounts of cesium chloride, using DMF as solvent, sodium (potassium) carboxylates reacted with $\alpha$-chloroacetates at room temperature or reacted with alkyl bromides (chlorides) at $60{ }^{\circ} \mathrm{C}$ to give the corresponding ( $\alpha$-alkoxyformacyl)methyl carboxylic esters and carboxylic esters in $>90 \%$ yields. The catalytic mechanism was discussed.
\end{abstract}

Keywords cesium chloride; sodium (potassium) carboxylates; alkyl bromides (chlorides); esterification

铯离子能活化与之结合的阴离子, 这是因为铯离子 体积大, 它与阴离子之间的静电作用较弱, 在极性的非 质子溶剂中, 铯离子易被溶剂化, 而由于溶剂偶极不易 靠近阴离子, 使阴离子不易被溶剂化而呈 “裸露” 的离 子. 导致与之键合的氢氧根离子表现出强碱性, 与之键 合的阴离子具有强的亲核能力. 碱金属氢氧化物碱性是 按 $\mathrm{LiOH}, \mathrm{NaOH}, \mathrm{KOH}, \mathrm{RbOH}, \mathrm{CsOH}$ 依次增强, 氢氧化 铯称之为超强碱 ${ }^{[1]}$, 它能与弱酸性的物质如端炔形成对 水稳定的炔负离子, 炔负离子能发生各种亲核反应 ${ }^{[1 \sim 3]}$; 阴离子具有强的亲核性, 可以降低反应活化能, 使需要 高温进行的反应在室温或温和条件下就能进行, 可有效 避免副反应发生, 达到提高反应选择性之目的. 根据上 述原理, 本课题组 ${ }^{[48]}$ 进行了铯离子催化端炔与二杂醚 反应研究.

酯在自然界的分布很广, 许多鲜花和水果的愉悦气
味和芳香均来源于酯. 很多药物的分子结构都含有酯 基. 酯的制备一般通过强酸催化下的羧酸与醇缩合，但 是大多数情况下需要加热, 有时需要高温. 所以这一方 法仅适合制备简单的酯，不适用于含有对酸或热敏感官 能团的底物. 通过羧酸盐与卤代烃反应是制备酯的另一 条途径. 羧酸盐亲核性弱, 一般只与活性高的卤代烃如 碘代烃或芐基卤反应，但碘代烃价格昂贵，导致其应用 受到限制; 对于活性低的澳或氯代烃, 与羧酸盐反应要 使用相转移催化剂, 且需要在高温条件下反应才能有效 进行 ${ }^{[9 \sim 13]}$. 我们根据铯离子活化阴离子原理, 设计用铯 离子催化羧酸盐与 $\alpha$-氯代乙酸酯和澳代烃反应制备相 应的 $\alpha$-烷氧甲酰甲酯与羧酸酯. 本文报道研究结果.

\section{1 结果与讨论}

羧酸盐与 $\alpha$-氯代乙酸酯反应制备 $\alpha$-烷氧甲酰甲酯.

*E-mail: xhx1581@yahoo.com.cn

Received August 13, 2012; revised September 8, 2012; published online September 21, 2012.

Project supported by the Natural Science Foundation of Hunan Province (No. 08JJ3018).

湖南省自然科学基金(No.08JJ3018)资助项目. 
以 $\alpha$-氯代乙酸苯甲酯与乙酸钠反应为模型, 考察氯化铯 对反应的影响. 实验表明, 不加氯化铯时, 乙酸钠 (1.0 $\mathrm{mmol})$ 和 $\alpha$-氯代乙酸苯甲酯 $(1.0 \mathrm{mmol}$ ) 在 $N, N$-二甲基甲 酰胺(DMF) (3.0 mL)中室温下反应 $10 \mathrm{~h}$, 产率仅为 $40 \%$. 加入 $5.0 \mathrm{~mol} \%$ 氯化铯 $(0.05 \mathrm{mmol})$, 室温下反应 $5.0 \mathrm{~h}$, 产 率 $62 \%$. 这结果表明氯化铯对这反应有明显的催化效 果. 继而考察 $\alpha$-氯代乙酸苯甲酯与乙酸钠, 氯化铯用量 与反应时间与产率之关系, 结果见表 1.

表 1 氯化铯用量与时间对 $\alpha$-氯代乙酸苯甲酯与乙酸钠反应 的影响

Table 1 Influence of amount of cesium chloride and time on the reaction of methyl $\alpha$-chloro-phenylacetate with sodium acetate

\begin{tabular}{cccc}
\hline Entry & $\mathrm{CsCl} / \mathrm{mol} \%$ & Time $/ \mathrm{h}$ & Yield $/ \%$ \\
\hline 1 & 5.0 & 5.0 & 62 \\
2 & 5.0 & 10.0 & 73 \\
3 & 5.0 & 20.0 & 81 \\
4 & 10.0 & 5.0 & 75 \\
5 & 10.0 & 10.0 & 82 \\
6 & 10.0 & 20.0 & 88 \\
7 & 20.0 & 5.0 & 91 \\
8 & 30.0 & 5.0 & 93 \\
\hline
\end{tabular}

由表 1 可知，随着氯化铯用量增加，反应速度增加; 在氯化铯用量一定情况下, 延长反应时间, 可提高产率. 当氯化铯用量为 $30.0 \mathrm{~mol} \%$, 与用量 $20.0 \mathrm{~mol} \%$ 相比, 产 率无明显变化. $20.0 \mathrm{~mol} \%$ 氯化铯的量是较佳选择. 在此 条件下, 考察其他羧酸盐与 $\alpha$-氯代乙酸酯反应(Eq. 1), 结果见表 2 .

由表 1 可知, 乙酸钠、苯甲酸钠、硬脂酸钾与不同 $\alpha$-氯代乙酸酯反应, 均取得高的收率. 取代基对反应有 一定影响. 羧酸盐及 $\alpha$-氯代乙酸酯中取代基 $\mathrm{R}^{1}$ 及 $\mathrm{R}^{2}$ 的 大小影响反应速度. 随着 $\mathrm{R}^{1}$ 及 $\mathrm{R}^{2}$ 的增大, 反应所需时 间增加.

$$
\begin{aligned}
& \mathrm{R}_{\mathbf{1}}^{1} \mathrm{COOM}+\underset{\mathbf{2}}{\mathrm{ClCH}_{2} \mathrm{COOR}^{2}} \frac{\mathrm{CsCl}(20 \mathrm{~mol} \%)}{\text { DMF, r.t. }} \\
& \mathrm{R}^{1} \mathrm{COOCH}_{2} \mathrm{COOR}^{2}+\mathrm{MX} \\
& 3 a \sim 3 h
\end{aligned}
$$

羧酸盐与澳代烃反应制备羧酸酯，以溴代正辛烷 $(1.0 \mathrm{mmol})$ 与苯甲酸钠 $(1.0 \mathrm{mmol})$ 反应为模型, 考察氯化 铯用量、温度及时间对反应的影响. 在没有氯化铯时, 室温下溴代正辛烷 $(1.0 \mathrm{mmol})$ 与苯甲酸钠 $(1.0 \mathrm{mmol})$ 在 无水 DMF (3.0 mL)中反应 $10 \mathrm{~h}$, 苯甲酸正辛酯产率仅为 $10 \%$; 反应 $24 \mathrm{~h}$, 产率为 $30 \%$; 加热到 $60{ }^{\circ} \mathrm{C}$, 反应 $10 \mathrm{~h}$ 后产率为 $40 \%$. 在氯化铯 $(10.0 \mathrm{~mol} \%, 0.10 \mathrm{mmol})$ 存在 下, 在室温下反应, 反应 $10 \mathrm{~h}$, 产率为 $35 \%$; 反应 $24 \mathrm{~h}$, 产率为 $66 \%, 60{ }^{\circ} \mathrm{C}$ 下反应 $10 \mathrm{~h}$, 产率达到 $83 \%$. 上述实 验表明, 催化剂用量及温度对反应有明显影响. 通过系 统探索, 得出较佳的反应条件是: 氯化铯用量为 20.0 $\mathrm{mol} \%$, 温度为 $60{ }^{\circ} \mathrm{C}$, 时间 $10.0 \mathrm{~h}$. 在此条件下, 考察多 种羧酸盐与溴代烃反应(Eq. 2), 结果见表 3.

$$
\underset{\mathbf{1}}{\mathrm{R}^{1} \mathrm{COOM}}+\underset{\mathbf{3}}{\mathrm{R}^{3} \mathrm{X}} \underset{\mathrm{DMF}, 60{ }^{\circ} \mathrm{C}}{\stackrel{\mathrm{CsCl}(20 \mathrm{~mol} \%)}{\mathbf{5 a} \sim 5 \mathbf{k}}} \underset{\mathrm{R}}{\mathrm{R}^{1} \mathrm{COOR}^{3}}+\mathrm{MBr}
$$

由表 2 知, 羧酸盐与活泼的芐澳反应, 在 $3.0 \mathrm{~h}$ 即可 达 $90 \%$ 产率; 羧酸盐与伯卤代烃的反应大部分能在 $10 \mathrm{~h}$ 能完成反应; 澳代烃比对应氯代反应快; 取代基越大， 反应速度越慢, 如硬脂酸钾与伯卤代烃反应需要较长反 应时间.

上述反应可能的机理如 Scheme 1.

$$
\begin{aligned}
& \mathrm{R}^{1} \mathrm{COOM}+\mathrm{CsCl} \rightleftharpoons \mathrm{RCOOCs}+\mathrm{MCl} \\
& \mathrm{R}^{1} \mathrm{COOCs}+\mathrm{R}^{3} \mathrm{Br} \rightleftharpoons \mathrm{RCOOR}+\mathrm{CsBr} \\
& \mathrm{CsBr}+\mathrm{RCOOM}=\mathrm{RCOOCs}+\mathrm{MBr}
\end{aligned}
$$

\begin{tabular}{|c|c|c|c|c|}
\hline Compd. & $\mathrm{R}^{1} \mathrm{COOM}$ & $\mathrm{ClCH}_{2} \mathrm{COOR}^{2}$ & Time/h & Isolated yield/\% \\
\hline $3 a$ & $\mathrm{CH}_{3} \mathrm{COONa}$ & $\mathrm{ClCH}_{2} \mathrm{COOCH}_{2} \mathrm{Ph}$ & 5.0 & 91 \\
\hline $\mathbf{3 b}$ & $\mathrm{CH}_{3} \mathrm{COONa}$ & $\mathrm{ClCH}_{2} \mathrm{COOCH}_{2} \mathrm{CH}_{2} \mathrm{Ph}$ & 5.0 & 93 \\
\hline $3 c$ & $\mathrm{CH}_{3} \mathrm{COONa}$ & & 10.0 & 92 \\
\hline 3d & $\mathrm{PhCOONa}$ & $\mathrm{ClCH}_{2} \mathrm{COOCH}_{3}$ & 5.0 & 95 \\
\hline $3 e$ & $\mathrm{PhCOONa}$ & $\mathrm{ClCH}_{2} \mathrm{COOCH}_{2} \mathrm{Ph}$ & 5.0 & 92 \\
\hline $3 f$ & $\mathrm{PhCOONa}$ & $\mathrm{ClCH}_{2} \mathrm{COOCH}_{2} \mathrm{CH}_{2} \mathrm{Ph}$ & 10.0 & 91 \\
\hline $3 g$ & $\mathrm{PhCOONa}$ & $\mathrm{CH}_{3}$ & 15.0 & 90 \\
\hline $3 \mathbf{h}$ & $\mathrm{CH}_{3}\left(\mathrm{CH}_{2}\right)_{15} \mathrm{CH}_{2} \mathrm{COOK}$ & $\mathrm{ClCH}_{2} \mathrm{COOCH}_{3}$ & 20.0 & 93 \\
\hline
\end{tabular}

\section{Scheme 1}

表 2 羧酸盐与 $\alpha$-氯代乙酸酯反应结果

Table 2 Reaction results of carboxylates and $\alpha$-chloroacetates 
表 3 羧酸盐与溴代烃反应结果

Table 3 Reaction results of carboxylates and alkyl bromides

\begin{tabular}{|c|c|c|c|c|}
\hline Compd. & $\mathrm{R}^{1} \mathrm{COOM}$ & $\mathrm{R}^{3} \mathrm{X}$ & Time/h & Isolated yield $/ \%$ \\
\hline $5 \mathbf{5 a}$ & $\mathrm{CH}_{3} \mathrm{COONa}$ & $\mathrm{PhCH}_{2} \mathrm{Br}$ & 3.0 & 90 \\
\hline $5 b$ & $\mathrm{CH}_{3} \mathrm{COONa}$ & $\mathrm{CH}_{3}\left(\mathrm{CH}_{2}\right)_{6} \mathrm{CH}_{2} \mathrm{Br}$ & 10.0 & 94 \\
\hline $5 c$ & $\mathrm{PhCOONa}$ & $\mathrm{CH}_{3} \mathrm{CH}_{2} \mathrm{CH}_{2} \mathrm{CH}_{2} \mathrm{Br}$ & 10.0 & 91 \\
\hline 5d & $\mathrm{PhCOONa}$ & $\mathrm{CH}_{3} \mathrm{CH}_{2} \mathrm{CH}_{2} \mathrm{CH}_{2} \mathrm{Cl}$ & 10.0 & 82 \\
\hline $5 e$ & $\mathrm{PhCOONa}$ & $\mathrm{CH}_{3} \mathrm{CH}_{2} \mathrm{CH}_{2} \mathrm{CH}_{2} \mathrm{Cl}$ & 15.0 & 90 \\
\hline $5 f$ & $\mathrm{PhCOONa}$ & $\mathrm{CH}_{3}\left(\mathrm{CH}_{2}\right)_{6} \mathrm{CH}_{2} \mathrm{Br}$ & 10.0 & 94 \\
\hline $5 g$ & $\mathrm{PhCOONa}$ & $\mathrm{PhCH}_{2} \mathrm{Br}$ & 3.0 & 90 \\
\hline $5 \mathrm{~h}$ & $\mathrm{CH}_{3}\left(\mathrm{CH}_{2}\right)_{15} \mathrm{CH}_{2} \mathrm{COOK}$ & $\mathrm{CH}_{3} \mathrm{CH}_{2} \mathrm{CH}_{2} \mathrm{CH}_{2} \mathrm{Br}$ & 20.0 & 90 \\
\hline $5 \mathbf{i}$ & $\mathrm{CH}_{3}\left(\mathrm{CH}_{2}\right)_{15} \mathrm{CH}_{2} \mathrm{COOK}$ & $\mathrm{CH}_{3}\left(\mathrm{CH}_{2}\right)_{6} \mathrm{CH}_{2} \mathrm{Br}$ & 20.0 & 91 \\
\hline $5 \mathbf{j}$ & $\mathrm{CH}_{3}\left(\mathrm{CH}_{2}\right)_{15} \mathrm{CH}_{2} \mathrm{COOK}$ & $\mathrm{PhCH}_{2} \mathrm{Br}$ & 8.0 & 92 \\
\hline $5 \mathrm{k}$ & $\mathrm{CH}_{2}=\mathrm{CH}\left(\mathrm{CH}_{2}\right)_{8} \mathrm{COOK}$ & $\mathrm{PhCH}_{2} \mathrm{CH}_{2} \mathrm{Br}$ & 18.0 & 92 \\
\hline
\end{tabular}

由 Scheme 1 可知, 羧酸钠或羧酸钾与氯化铯通过 离子交换产生亲核性高的羧酸铯, 羧酸铯与溴代烃反应 形成羧酸酯及溴化铯, 溴化铯随后再与羧酸盐进行离子 交换产生羧酸铯.

\section{2 结论}

本文研究工作表明, 催化量氯化铯可以加速羧酸盐 与卤代烃反应, 提高产率. 本方法为改善羧酸酯的制备 提供了一条新途径.

\section{3 实验部分}

\section{1 仪器与试剂}

${ }^{1} \mathrm{H}$ NMR(以 TMS 为内标), ${ }^{13} \mathrm{C}$ NMR(以 TMS 为内 标)用 INOVA-400 型仪测定, 质谱由 HP5989A 测定. DMF 用氢化钙干燥, 硅胶为青岛海洋化工厂产品.

\section{2 实验方法}

3.2.1 羒酸盐与 $\alpha$-氯代乙酸酯反应合成 $\alpha$-烷氧甲酰 甲酯

在圆底烧瓶瓶中依次加入羒酸盐 $(1.0 \mathrm{mmol})$, 无水 DMF $(3.0 \mathrm{~mL}), \alpha$-氯代乙酸酯 $(1.0 \mathrm{mmol})$, 氯化铯 $(0.2$ $\mathrm{mmol})$, 在室温摚拌, 反应时间见表 1 . 反应后减压蒸出 溶剂, 加入 $50 \mathrm{~mL}$ 石油醚稀释, 水洗, 无水硫酸钠干燥, 用石油醚/乙醚 $(V: V=30: 1)$ 作洗脱剂进行柱层析纯 化.

乙酸 $\left(\alpha\right.$-苯甲氧甲酰)甲酯 $(3 a)^{[19]}$ : 浅黄色油状液体; ${ }^{1} \mathrm{H}$ NMR (400 MHz, $\left.\mathrm{CDCl}_{3}\right) \delta: 2.13$ (s, 3H), 4.43 (s, 2H), $4.63(\mathrm{~s}, 2 \mathrm{H}), 7.32 \sim 7.36(\mathrm{~m}, 5 \mathrm{H}) ;{ }^{13} \mathrm{C}$ NMR $(100 \mathrm{MHz}$, $\left.\mathrm{CDCl}_{3}\right) \delta: 170.3,169.1,136.1,128.9,127.1,66.1,60.4$, 20.4; MS (EI) $m / z: 208\left(\mathrm{M}^{+}\right)$.

乙酸 $\left(\alpha\right.$-苯乙氧甲酰)甲酯 $(3 \mathbf{b})^{[14]}$ : 浅黄色油状液体. ${ }^{1} \mathrm{H}$ NMR (400 MHz, $\mathrm{CDCl}_{3}$ ) $\delta: 2.13$ (s, 3H), 2.95 (t, $J=8.0$ $\mathrm{Hz}, 2 \mathrm{H}), 4.37$ (t, $J=8.0 \mathrm{~Hz}, 2 \mathrm{H}), 4.57$ (s, 2H), $7.19 \sim 7.32$ (m, 5H); ${ }^{13} \mathrm{C}$ NMR (100 MHz, $\left.\mathrm{CDCl}_{3}\right) \delta: 170.5,169.4$, 138.5, 128.7, 127.6, 125.9, 64.7, 60.4, 34.6, 20.3; MS (EI) $m / z: 222\left(\mathrm{M}^{+}\right)$.

乙酸 $\left[\left(3,7\right.\right.$-二甲基-2,6-二烯)辛氧甲酰]甲酯 $(3 \mathbf{c})^{[14]}$ : 浅黄色油状液体. ${ }^{1} \mathrm{H}$ NMR (400 MHz, CDCl3) $\delta: 1.60$ $1.71(\mathrm{~m}, 9 \mathrm{H}), 2.16(\mathrm{~s}, 3 \mathrm{H}), 2.04 \sim 2.09(\mathrm{~m}, 4 \mathrm{H}), 4.60(\mathrm{~s}$, $2 \mathrm{H}), 4.68(\mathrm{~d}, J=8 \mathrm{~Hz}, 2 \mathrm{H}), 5.06 \sim 5.09(\mathrm{~m}, 1 \mathrm{H}), 5.31 \sim$ $5.35(\mathrm{~m}, 1 \mathrm{H}) ;{ }^{13} \mathrm{C}$ NMR $\left(100 \mathrm{MHz}, \mathrm{CDCl}_{3}\right) \delta: 170.3$, 167.1, 132.0, 129.5, 123.5, 120.0, 61.7, 35.9, 26.3, 24.6, 20.4, 18.6, 15.5; MS (EI) $m / z: 240\left(\mathrm{M}^{+}\right)$.

苯甲酸 $\left(\alpha\right.$-甲氧甲酰)甲酯 $(\mathbf{3 d})^{[15]}$ : 黄色油状液体. ${ }^{1} \mathrm{H}$ NMR (400 MHz, $\left.\mathrm{CDCl}_{3}\right) \delta: 3.78(\mathrm{~s}, 3 \mathrm{H}), 4.86(\mathrm{~s}, 2 \mathrm{H})$, $7.43 \sim 8.10(\mathrm{~m}, 5 \mathrm{H}) ;{ }^{13} \mathrm{C}$ NMR $\left(100 \mathrm{MHz}, \mathrm{CDCl}_{3}\right) \delta$ : $166.9,165.7,133.1,130.3,129.8,128.5,61.2,51.6$; MS (EI) $m / z: 194\left(\mathrm{M}^{+}\right)$.

苯甲酸 $\left(\alpha\right.$-苯甲氧甲酰)甲酯 $(3 \mathbf{e})^{[16]}$ : 黄色油状液体. ${ }^{1} \mathrm{H}$ NMR $\left(400 \mathrm{MHz}, \mathrm{CDCl}_{3}\right) \delta: 4.89$ (s, 2H), 5.23 (s, 2H) $7.36 \sim 8.11(\mathrm{~m}, 10 \mathrm{H}) ;{ }^{13} \mathrm{C}$ NMR $\left(100 \mathrm{MHz}, \mathrm{CDCl}_{3}\right) \delta$ : $169.3,167.2,138.8,136.1,133.4,128.9,127.6,127.1$, 126.8, 66.2, 63.5; MS (EI) $m / z: 270\left(\mathrm{M}^{+}\right)$.

苯甲酸 $\left(\alpha\right.$-苯乙氧甲酰)甲酯 $(\mathbf{3 f})^{[16]}$ : 黄色油状液体. ${ }^{1} \mathrm{H}$ NMR $\left(400 \mathrm{MHz}, \mathrm{CDCl}_{3}\right) \delta: 2.95(\mathrm{t}, J=8.0 \mathrm{~Hz}, 2 \mathrm{H})$, $4.38 \sim 4.41(\mathrm{t}, J=8.0 \mathrm{~Hz}, 2 \mathrm{H}), 4.83(\mathrm{~s}, 2 \mathrm{H}), 7.17 \sim 8.09$ $(\mathrm{m}, 10 \mathrm{H}) ;{ }^{13} \mathrm{C}$ NMR $\left(100 \mathrm{MHz}, \mathrm{CDCl}_{3}\right) \delta: 169.4,165.8$, 138.2, 133.0, 130.1, 129.9, 128.6, 128.5, 127.7, 125.8, 64.9, 61.5, 34.5; MS (EI) $m / z: 284\left(\mathrm{M}^{+}\right)$.

苯甲酸 [(3,7-二甲基-2,6-二烯) 辛氧甲酰]甲酯 $(3 \mathrm{~g})^{[17]}$ : 黄色油状液体. ${ }^{1} \mathrm{H}$ NMR $\left(400 \mathrm{MHz}, \mathrm{CDCl}_{3}\right) \delta$ : $1.60 \sim 1.70(\mathrm{~m}, 9 \mathrm{H}), 2.04 \sim 2.10(\mathrm{~m}, 4 \mathrm{H}), 4.72(\mathrm{~d}, J=8 \mathrm{~Hz}$, 2H), $4.85(\mathrm{~s}, 2 \mathrm{H}), 5.06(\mathrm{t}, J=6.0 \mathrm{~Hz}, 1 \mathrm{H}), 5.35$ (t, $J=6.0$ $\mathrm{Hz}, 1 \mathrm{H}), 7.26 \sim 8.11(\mathrm{~m}, 5 \mathrm{H}) ;{ }^{13} \mathrm{C}$ NMR $(100 \mathrm{MHz}$, $\left.\mathrm{CDCl}_{3}\right) \delta: 167.1,165.8,133.0,132.0,130.1,129.9,129.7$, $128.5,123.5,120.1,61.6,35.9,26.1,24.6,18.6,12.5$; MS 
(EI) $m / z: 302\left(\mathrm{M}^{+}\right)$.

硬脂酸 $\left(\alpha\right.$-甲氧甲酰)甲酯 $(3 \mathbf{h}){ }^{[17]}$ : 黄色粘稠液体. ${ }^{1} \mathrm{H}$ NMR (400 MHz, $\left.\mathrm{CDCl}_{3}\right) \delta: 0.88(\mathrm{t}, J=6.0 \mathrm{~Hz}, 3 \mathrm{H})$, $1.25 \sim 1.68(\mathrm{~m}, 28 \mathrm{H}), 2.42(\mathrm{t}, J=6.0 \mathrm{~Hz}, 2 \mathrm{H}), 3.77(\mathrm{~s}, 3 \mathrm{H})$, $4.62(\mathrm{~s}, 2 \mathrm{H}) ;{ }^{13} \mathrm{C}$ NMR (100 MHz, $\left.\mathrm{CDCl}_{3}\right) \delta: 173.1,166.7$, 60.4, 51.6, 33.7, 29.6, 29.3, 29.0, 25.1, 21.4, 14.1; MS (EI) $m / z: 356\left(\mathrm{M}^{+}\right)$.

3.2 .2 羧酸盐与 $\alpha$-氯代乙酸酯反应合成 $\alpha$-烷氧甲酰 甲酯

在圆底烧瓶瓶中依次加入羧酸盐 $(1.0 \mathrm{mmol})$, 无水 DMF (3.0 mL), 溴代烃 $(1.0 \mathrm{mmol})$, 氯化铯 $(0.2 \mathrm{mmol})$, 在 $60{ }^{\circ} \mathrm{C}$ 摚拌, 反应时间见表 2 . 反应后减压蒸出溶剂, 加入石油醚 $(50 \mathrm{~mL})$ 稀释, 水洗, 无水硫酸钠干燥, 用石 油醚/乙醚 $(V: V=30: 1)$ 作洗脱剂进行柱层析纯化.

乙酸苯甲酯 $(\mathbf{5 a})^{[18]}$ : 油状液体. ${ }^{1} \mathrm{H}$ NMR $(400 \mathrm{MHz}$, $\left.\mathrm{CDCl}_{3}\right) \delta: 2.08$ (s, 3H), 5.09 (s, 2H), $7.30 \sim 7.35$ (m, 5H); ${ }^{13} \mathrm{C}$ NMR $\left(100 \mathrm{MHz}, \mathrm{CDCl}_{3}\right) \delta: 170.3,136.2,128.8,127.4$, 127.2, 66.4, 20.3; MS (EI) $m / z: 150\left(\mathrm{M}^{+}\right)$.

乙酸正辛酯 $(\mathbf{5 b})^{[19]}$ : 油状液体. ${ }^{1} \mathrm{H}$ NMR $(400 \mathrm{MHz}$, $\left.\mathrm{CDCl}_{3}\right) \delta: 0.88(\mathrm{t}, J=6.0 \mathrm{~Hz}, 3 \mathrm{H}), 1.28 \sim 1.36(\mathrm{~m}, 10 \mathrm{H})$, $1.60 \sim 1.63(\mathrm{~m}, 2 \mathrm{H}), 2.05(\mathrm{~s}, 3 \mathrm{H}), 4.05(\mathrm{t}, J=6.0 \mathrm{~Hz}, 2 \mathrm{H})$; ${ }^{13} \mathrm{C} \mathrm{NMR}\left(100 \mathrm{MHz}, \mathrm{CDCl}_{3}\right) \delta: 170.1,65.4,30.2,29.1$, 28.6, 25.4, 22.6, 20.5, 14.3; MS (EI) $m / z: 172\left(\mathrm{M}^{+}\right)$.

苯甲酸正丁酯 $(\mathbf{5 c})^{[19]}$ : 油状液体. ${ }^{1} \mathrm{H}$ NMR (400 $\left.\mathrm{MHz}, \mathrm{CDCl}_{3}\right) \delta: 0.98(\mathrm{t}, J=6.4 \mathrm{~Hz}, 3 \mathrm{H}), 1.48 \sim 1.54(\mathrm{~m}$, $2 \mathrm{H}), 1.76 \sim 1.83(\mathrm{~m}, 2 \mathrm{H}), 4.33(\mathrm{t}, J=6.4 \mathrm{~Hz}, 2 \mathrm{H}), 7.26 \sim$ $8.06(\mathrm{~m}, 5 \mathrm{H}) ;{ }^{13} \mathrm{C} \mathrm{NMR}\left(100 \mathrm{MHz}, \mathrm{CDCl}_{3}\right) \delta: 167.1$, $133.6,130.4,129.8,128.4,64.7,31.4,18.5,14.4$; MS (EI) $m / z: 178\left(\mathrm{M}^{+}\right)$.

苯甲酸正辛酯 $(\mathbf{5 f})^{[20]}$ : 油状液体. ${ }^{1} \mathrm{H}$ NMR (400 $\left.\mathrm{MHz}, \mathrm{CDCl}_{3}\right) \delta: 0.88(\mathrm{t}, J=6.4 \mathrm{~Hz}, 3 \mathrm{H}), 1.28 \sim 1.46(\mathrm{~m}$, $10 \mathrm{H}), 1.73 \sim 1.76(\mathrm{~m}, 2 \mathrm{H}), 4.31(\mathrm{t}, J=6.4 \mathrm{~Hz}, 2 \mathrm{H}), 7.48 \sim$ $8.06(\mathrm{~m}, 5 \mathrm{H}) ;{ }^{13} \mathrm{C} \mathrm{NMR}\left(100 \mathrm{MHz}, \mathrm{CDCl}_{3}\right) \delta: 165.8$, 132.9, 130.1, 129.6, 128.5, 64.5, 31.9, 30.6, 29.7, 25.6, 22.4, 14.2; MS (EI) $m / z: 234\left(\mathrm{M}^{+}\right)$.

苯甲酸苯甲酯 $(\mathbf{5 g})^{[20]}$ : 浅黄色油状液体. ${ }^{1} \mathrm{H}$ NMR $\left(400 \mathrm{MHz}, \mathrm{CDCl}_{3}\right) \delta: 5.09$ (s, 2H), $7.39 \sim 8.09$ (m, 10H); ${ }^{13} \mathrm{C}$ NMR (100 MHz, $\left.\mathrm{CDCl}_{3}\right) \delta: 167.3,136.5,133.7,130.5$, 129.7, 128.7, 128.3, 127.6, 127.1, 65.5; MS (EI) $\mathrm{m} / \mathrm{z}: 212$ $\left(\mathrm{M}^{+}\right)$.

硬脂酸正丁酯 $(\mathbf{5 h})^{[21]}$ : 浅黄色油状液体. ${ }^{1} \mathrm{H}$ NMR $\left(400 \mathrm{MHz}, \mathrm{CDCl}_{3}\right) \delta: 0.88(\mathrm{t}, J=6.0 \mathrm{~Hz}, 3 \mathrm{H}), 0.93(\mathrm{t}, J=$ $6.2 \mathrm{~Hz}, 3 \mathrm{H}), 1.25 \sim 1.39(\mathrm{~m}, 30 \mathrm{H}), 1.59 \sim 1.63(\mathrm{~m}, 4 \mathrm{H})$, 2.29 (t, $J=6.4 \mathrm{~Hz}, 2 \mathrm{H}), 4.07$ (t, $J=6.4 \mathrm{~Hz}, 2 \mathrm{H}) ;{ }^{13} \mathrm{C} \mathrm{NMR}$ $\left(100 \mathrm{MHz}, \mathrm{CDCl}_{3}\right) \delta: 173.3,64.8,33.7,31.1,29.6,29.3$,
29.0, 25.0, 22.6, 18.7, 14.3, 13.7; MS (EI) m/z: $340\left(\mathrm{M}^{+}\right)$.

硬脂酸正辛酯 $(\mathbf{5 i})^{[21]}$ : 浅黄色油状液体. ${ }^{1} \mathrm{H}$ NMR $\left(400 \mathrm{MHz}, \mathrm{CDCl}_{3}\right) \delta: 0.86 \sim 0.90(\mathrm{~m}, 6 \mathrm{H}), 1.25 \sim 1.28(\mathrm{~m}$, $40 \mathrm{H}), 1.60 \sim 1.63(\mathrm{~m}, 4 \mathrm{H}), 2.29(\mathrm{t}, J=6.4 \mathrm{~Hz}, 2 \mathrm{H}) ;{ }^{13} \mathrm{C}$ NMR (100 MHz, $\left.\mathrm{CDCl}_{3}\right) \delta: 173.1,65.2,33.6,31.5,29.6$, 29.3, 29.0, 28.7, 25.8, 25.1, 22.7, 18.7, 14.1; MS (EI) $m / z$ : $396\left(\mathrm{M}^{+}\right)$.

硬脂酸苯甲酯 $(\mathbf{5 j})^{[22]}$ : 浅黄色油状液体. ${ }^{1} \mathrm{H}$ NMR $\left(400 \mathrm{MHz}, \mathrm{CDCl}_{3}\right) \delta: 0.88(\mathrm{t}, J=6.4 \mathrm{~Hz}, 3 \mathrm{H}), 1.25 \sim 1.28$ $(\mathrm{m}, 28 \mathrm{H}), 1.62 \sim 1.65(\mathrm{~m}, 4 \mathrm{H}), 2.35(\mathrm{t}, J=6.4 \mathrm{~Hz}, 2 \mathrm{H})$, $5.11(\mathrm{~s}, 2 \mathrm{H}), 7.26 \sim 7.38(\mathrm{~m}, 5 \mathrm{H}) ;{ }^{13} \mathrm{C} \mathrm{NMR}(100 \mathrm{MHz}$, $\left.\mathrm{CDCl}_{3}\right) \delta: 173.2,136.4,128.8,127.6,127.1,66.2,33.8$, $31.7,29.6,29.3,29.0,25.1,22.6,14.1$; MS (EI) $\mathrm{m} / \mathrm{z}: 374$ $\left(\mathrm{M}^{+}\right)$.

11-烯十一酸(2-苯基)乙酯 $(\mathbf{5 k})^{[22]}$ : 浅黄色油状液体. ${ }^{1} \mathrm{H}$ NMR $\left(400 \mathrm{MHz}, \mathrm{CDCl}_{3}\right) \delta: 1.27 \sim 1.33(\mathrm{~m}, 10 \mathrm{H})$, $1.36 \sim 1.40(\mathrm{~m}, 2 \mathrm{H}), 1.58 \sim 1.65(\mathrm{~m}, 2 \mathrm{H}), 2.04(\mathrm{dt}, J=1.2$, $8.0 \mathrm{~Hz}, 2 \mathrm{H}), 2.27(\mathrm{t}, J=7.6 \mathrm{~Hz}, 2 \mathrm{H}), 2.93(\mathrm{t}, J=7.2 \mathrm{~Hz}$, $2 \mathrm{H}), 4.38$ (t, $J=7.2 \mathrm{~Hz}, 2 \mathrm{H}), 4.94$ (dd, $J=1.2,10.4 \mathrm{~Hz}$, $1 \mathrm{H}), 4.99$ (dd, $J=1.6,17.2 \mathrm{~Hz}, 1 \mathrm{H}), 5.81 \sim 5.88(\mathrm{~m}, 1 \mathrm{H})$, $7.20 \sim 7.24(\mathrm{~m}, 3 \mathrm{H}), 7.28 \sim 7.32(\mathrm{~m}, 2 \mathrm{H}) ;{ }^{13} \mathrm{C}$ NMR $(100$ $\left.\mathrm{MHz}, \mathrm{CDCl}_{3}\right) \delta: 174.5,139.8,138.6,128.3,127.5,125.7$, $115.6,65.4,33.9,34.4,29.8,29.3,28.7,25.1$; MS (EI) $m / z$ : $288\left(\mathrm{M}^{+}\right)$.

\section{References}

[1] Tzalis, D.; Knochel, P. Angew. Chem., Int. Ed. 1999, 38, 1463.

[2] Salvatore, R. N.; Nagle, A. S.; Jung, K. W. J. Org. Chem. 2002, 67, 674.

[3] Tzalis, D.; Koradin, C.; Knochel, P. Tetrahedron Lett. 1999, 40, 6193.

[4] Li, X. W.; Liu, W. Q.; Zou, K. B. Chin. J. Org. Chem. 2007, 27, 1176 (in Chinese). (黎小武, 刘文奇, 邹康兵, 许新华, 有机化学, 2007, 27, 1176.)

[5] Xia, X.; Zou, K. B.; Fang, D. W.; Xu, X. H. Chin. J. Org. Chem. 2008, 28, 1487 (in Chinese). (夏湘, 邹康兵, 方大为, 许新华, 有机化学, 2008, 28, 1487.)

[6] Zou, K. B.; Qiu, R. H.; Fang, D. W.; Liu, X. Y.; Xu, X. H. Synth. Commun. 2008, 38, 237.

[7] Zou, K. B.; Yin, X. H.; Liu, W. Q.; Qiu, R. H.; Li, L. X.; Shao, L. L.; Li, Y. H.; Xu, X. H.; Yang, R. H. Synth. Commun. 2009, 39, 646.

[8] Xia, X.; Zou, K. B.; Li, N. X.; Zhang, Z. Y.; Shao, L. L.; Xu, X. H. Acta Chim. Sinica 2008, 66, 1749. (夏湘, 邹康兵, 李诺信, 张尊英, 郡玲玲, 许新华, 化学学报, 2008, 66, 1749.)

[9] Steven, C. W.; Rayman, Y. W. Tetrahedron Lett. 1972, 13, 1853.

[10] Douglas, J. R.; Patrick, G.; Albert, O. B. J. Org. Chem. 1979, 44, 1149.

[11] Zaher, M. A. J.; Hao, Y. S.; Bun, C. C. Tetrahedron Lett. 2002, 43, 9381.

[12] Cheng, B. C.; Xie, W. L. J. Central South Univ. Technol. 1998, 29, 
401 (in Chinese).

(成本诚, 谢文林, 中南工业大学学报, 1998, 29, 401.)

[13] Chen, D. M.; Yang, H. R.; Li, B. N. Petrochem. Technol. 1999, 28, 528 (in Chinese).

(陈达美, 杨辉荣, 黎碧娜, 石油化工, 1999, 28, 5.)

[14] Barratt, B. J. W.; Easton, C. J.; Simpson, J. S. PCT Int. Appl. 2004, $47,87$.

[15] Nielsen, N. M.; Bundgaard, H. Int. J. Pharm. 1987, 391, 75.

[16] Murai, K.; Akazome, G. Kogyo Kagaku Zasshi 1959, 62, 1094.

[17] Liu, Y.; Wei, M.; Liu, Z.-D. China Food Additives 2011, 4, 8.
[18] Sadieva, N. F.; Iskenderova, S. A.; Agaev, B. K.; Magerramova, S. N. Azerb. Khim. Zh. 2010, (4), 68.

[19] Nudelman, N. S.; Mendiara, S. Tetrahedron Lett. 1997, 38(13), 2245.

[20] Long, L.-P.; Zhong, T.-S.; Ding, L.-Z.; Yu, S.-X. Chin. J. Synth. Chem. 2002, 10(6), 542.

[21] Vijayakumar, B.; Nagendrappa, G.; Prakash, B. S. J. Indian J. Chem. Technol. 2009, 16(5), 377.

[22] Zhang, M.; Hanson, P. R. Sci. Synth. 2006, 20, 863.

(Li, L.; Lu, Z.) 